\title{
EVALUATION OF PLASMA LACTATE CONCENTRATION IN UNEXPLAINED MYOPATHIES WITH MULTI-ORGAN INVOLVEMENT
}

Papia Mondal, Animesh Kar.

1. Demonstrator. Department of Biochemistry, Bankura Samnilani Medical College.

2. Consultant. Department of Neurologist, Rabindranath Tagore International Institute of Cardiac Sciences, Kolkata.

\section{CORRESPONDING AUTHOR}

Dr. Papia Mondal.

N/365, B.P. Township,

Kolkata- 94.

E-mail: karanidoc@gmail.com

Ph: 00919830216167.

ABSTRACT: Myopathies are disorders of muscle with structural changes or functional impairment. Mitochondrial myopathies are caused by genetic mutations that directly influence the functioning of the electron transport chain. The ubiquitous distribution of the mitochondria in the human body explains the multiple organ involvement. Diagnosis often requires a multifaceted approach with measurements of serum lactate, magnetic resonance spectroscopy, muscle histology and ultrastructure, enzymology, genetic analysis, and exercise testing. The present study was conducted to find out the reason behind unexplained myopathies with multiorgan involvement by measuring plasma lactate concentration in patients who were suspected to be suffering from mitochondrial disorders. Muscle biopsy samples of those patients were also subjected for histopathological assessment. Out of twenty suspected patients, nine patients showed high lactate concentration and their muscle biopsy sample also revealed some changes suggestive of mitochondrial disorders.

KEYWORDS: mitochondria, lactate, myopathy.

INTRODUCTION: Myopathies are disorders of muscle with structural changes or functional impairment. It is differentiated from other diseases of motor unit by characteristic clinical and laboratory findings. There are a large number of myopathies where the cause can be ascertained like hereditary myopathies ( Duchenne muscular dystrophy, Becker muscular dystrophy, Facioscapulohumeral muscular dystrophy, etc), congenital myopathies like central core myopathy, nemaline myopathy, and myopathy due to disorders of muscle energy metabolism ( like disorders due to glycogen storage causing progressive weakness). ${ }^{1}$

But still there exists a group of myopathies where the cause of myopathy is not easily identifiable. This unexplained group of myopathies is often associated with abnormalities of other systems. A significant percentage of this latter group may comprise of myopathies due to mitochondrial disorders that result from mutation in mitochondrial DNA ( mtDNA) or nuclear DNA ( $\mathrm{nDNA}$ ) coding for mitochondrial proteins. ${ }^{2}$ There are various types of clinical presentations of this group of myopathies because of large number of reported mutations in both mtDNA and nDNA along with phenomenon like heteroplasmy, mitotic segregation and maternal inheritance.,4 Several studies carried out elsewhere have indicated that the minimum prevalence of mitochondrial disorders in myopathies is at least 1 in 5,000 and could be much higher, 5, 6, 7, 8 but data on this topic is not available in our population. Because of presence of 
large number of mutations in such patients, it may not be realistic to screen for suspected mitochondrial disorders of uncertain origin routinely by genetic analysis. For such reason biochemical assessment with histopathological tests can be of immense value from the diagnostic point of view. Hence this study was conducted for biochemical screening for suspected mitochondrial disorders by measuring the plasma levels of lactate and histopathological tests of muscle biopsy sample.

MATERIALS AND METHODS: Study population included myopathy patients attending the neuromedicine out-patient department and indoor of a tertiary care hospital in Kolkata from 2007-2009.

\section{INCLUSION CRITERION:}

1. Patients who had weakness suggestive of myopathy like predominantly proximal weakness, preserved or hypertrophied extensor digitorum brevis, with normal or decreased deep tendon reflexes, with normal sensation with normal sphincter function were included.

2. Patients also having one or many of cognitive decline, ataxia, ptosis with no history of diplopia, diabetes mellitus, seizure disorder, deafness, cardiac abnormality.

Patients who had only weakness suggestive of myopathy were excluded from the study.

Plasma lactate estimation was done from blood collected in sodium fluoride vial from a stasis free vein from a patient fasting for 12 hours and at complete rest and stored in an ice bath. The plasma is then separated by centrifugation within 30 minutes. Any delay would have led to a rise in lactate value. Assay was done immediately using a commercial kit based on the principle of lactate oxidase method (Randox kit)

The concentration of L-lactate in the sample is determined according to the following equation:

L-lactate + Oxygen $\quad$ Lactate oxidase $\rightarrow$ Pyruvate + Hydrogen peroxide.

Peroxidase

Hydrogen peroxide + TOSS -------------------- Purple product + H2O.

TOSS: $\mathrm{N}$ methyl - $\mathrm{N}$ - (2 hyroxy-3 sulphopropyl) m- toluidine.

Muscle biopsy sample collection was done from vastus lateralis muscle under local anesthesia (about 0.5 to 1 gram of muscle tissue was collected) in formalin and brought to the laboratory for histopathological assessment.

RESULT AND ANALYSIS: In this study, plasma lactate estimation and muscle biopsy was taken from 20 patients who are strongly suspected to have mitochondrial disorder on clinical basis. From twenty otherwise healthy subjects who had no symptoms included in the inclusion criterion plasma lactate was also estimated which we considered as our control value. Histopathological examination was done in our institute and finding in patients with 
serial number 1-9 were suggestive of mitochondrial myopathy (muscle fibre caliber variability, fibre type specific atrophy, necrosis and regeneration of myofibrils).

DISCUSSION: Till relatively recently mitochondrial disorders were considered to be obscure and exceptionally rare disease affecting perhaps 1-2 per million of the population. But different epidemiological studies done outside India clearly demonstrates that mitochondrial disorders are among the most common inherited human diseases. ${ }^{9}$ The diagnosis of mitochondrial disorders is a hugely challenging problem. Clinical presentations are varied but usually patients present with manifestations of neurological and muscular disorders ranging from impaired vision, ptosis, deafness, seizures, stroke, muscle weakness, atrophy, gait impairment, diabetes mellitus, gastropersis, and etc. 10

The diagnosis may be confused with inflammatory myopathies, muscular dystrophies, spino-cerebeller ataxia, limb- girdle atrophy, etc. ${ }^{11}$ Routine biochemical investigations of blood or serum, electrocardiography, echocardiography and special investigations like, electromyography, electroencephalography, CT scan Brain, MRI Brain often are not able to click the diagnosis. Any multi-system progressive disorder should lead a physician to suspect a mitochondrial disorder. ${ }^{12} \mathrm{~A}$ diagnosis can be particularly difficult if there is only one symptom. The diagnostic criterion for mitochondrial myopathies involves phenotypic evaluation or evaluation of observable traits, followed by laboratory evaluation. ${ }^{11} \mathrm{~A}$ clinical diagnosis can be confirmed by laboratory studies, muscle biopsy and molecular genetic evaluation in which a geneticist analyses the mt DNA.13, 14. If an mtDNA mutation is detected, a diagnosis is much more straightforward. In the absence of an mt DNA mutation, diagnosis becomes difficult.12 $\mathrm{A}$ disturbance at any step in oxidative phosphorylation would cause a rise in mitochondrial $\mathrm{NADH} / \mathrm{NAD}+$ ratio (redox state) which in turn result in increase in lactate concentration. ${ }^{11,15}$, 16.Different types of histopathological abnormalities were found in muscle biopsies from patients with mitochondrial diseases. Such as muscle fibre caliber variability, fibre type specific atrophy, necrosis and regeneration of myofibrils and inter-myofibrillar accumulation of lipids or glycogens. Peripheral nerve involvement is very common in mitochondrial diseases. Muscle biopsy often show partial denervation. ${ }^{11}$ The hallmark feature in mitochondrial disease is "ragged red" fibres (RRF). These are sub-sarcolemmal and myofibrillar accumulation of mitochondria and appears as bright red masses on modified Gomori Trichrome stain. 11, 17, 18.These abnormal accumulations represent a compensatory proliferation of mitochondria, some of which are ultra-structurally normal and others are dystrophic. ${ }^{11,19 .}$

In our study, out of 20 suspected cases of mitochondrial disorders plasma lactate are significantly high in nine subjects. \{Table 1\} The remaining eleven suspected cases of mitochondrial disorders have been proved to be suffering from other neuro-muscular disorders like polymyositis, muscular dystrophy as verified from histo-pathological and other investigations. Histopathological investigation of muscle biopsy sample of these nine subjects also revealed some features of mitochondrial disorders like muscle fibre caliber variability, fibre type specific atrophy, necrosis and regeneration of myofibrils. And in two of our patients there was also presence of "ragged red" fibres (RRF). This indicates a high degree of specificity of plasma lactate value along with histopathological assessment of mitochondrial disorders. Nine suspected cases that had been diagnosed as patients of mitochondrial myopathies however were not subjected to genetic testing because already greater then 150 mutations have been identified and it is not feasible to do all types of mutation analyses. ${ }^{20,} 21$ But biochemical analysis of the respiratory chain enzymes in muscles of the patients included in our study is in progress which will further confirm the diagnosis of mitochondrial disorders in the future. 
However the present study has some limitations because of the limited sample size for controls and diseased subjects and tissue specific defects that only affect the brain may not be detected in skeletal muscle. ${ }^{12}$

CONCLUSION: This present study has shown that assessment of plasma lactate concentration followed by histopathological examination can be an effective method for the diagnosis of mitochondrial disorders who present with myopathy and other organ involvement. However biochemical analysis of the respiratory chain enzymes in muscles is in progress which will further confirm the diagnosis of mitochondrial disorders. And this may replace the need for mutational analyses to confirm the diagnosis of mitochondrial disorder.

\section{REFERENCES:}

1. Jerry R. Mendell,Harrison's Principles of Internal Medicine, vol 11: 16: 2524.

2. DiMauro S,Mitochondrial Myopathy,Curr Opin Rheumatol 2006: 18: 636-641.

3. DiMauro S, Davidzon G, Mitochondrial DNA and disease, Annals of Medicine, 2005: 37: 222-232.

4. DiMauro S, Hirano M, Schon EA, editors. Mitochondrial medicine. London: informa Healthcare: 2006: 636-641.

5. Chinnery PF, Wardell TM, Singh-Kler R, Hayes C, Johnson MA, Taylor RW, et al. Ann Neurol. 2000: 48: 188-93.

6. Schaefer AM, Taylor RW, Turnbull DM, Chinnery PF. The epidemiology of mitochondrial disorders- past, present, and fyture. BBA. 2004: 1659: 115-120.

7. Darin N, Oldfors A, Moslemi AR, Holme E, Tulinius M. The incidence of mitochondrial encephalomyopathies in childhood: clinical features and morphological, biochemical and DNA abnormalities. Ann Neurol. 2001: 49: 377-383.

8. Skladal D, Halliday J, Thorburn DR. Brain. 2003: 126: 1905-12.

9. Mcmillan $\mathrm{C}$, Kirkham $\mathrm{T}$, et al. Pedigree analysis of fFrench Canadian families with T14484C LHON. Neurology. 1998: 50: 417-422.

10. Lowry OH, Rosenbrough NJ, Farr AL, Randall RJ. Protein measurement with the Folin phenol reagent. J Biol Chem. 1951 193: 265-275.

11. Neurology in Clinical Practice. Walter J Bradley: $5^{\text {th }}$ edition: $1785-1798$.

12. Brain's diseases of Nervous System. Edited by Michael Donghly: $12^{\text {th }}$ edition.

13. Clavio S, Jain M, Xie X, Sheth SA, Chang B, Goldberger OA, Spinazzola A, Zeviani M, Carr SA, Mootha VK. Systematic identification of human mitochondrial disease genes through integrative genomics. Nature Genet 2006: 38: 576-582.

14. Crimi M O, Hearn SF, Wallace DC, Gomi CP. Molecular research technologies in mitochondrial diseases: the microarray approach. IUBMB Life 2005: 57: 811-818.

15. Pulkes T, Eunson L, Patterson V, Siddique A, Wood NW, Nelson IP et al. The mitochondrial DNA G13513A transition in ND5 is associated with a LHON/MELAS overlap syndrome and may be a frequent cause.

16. Corona $\mathrm{P}$, et al. A novel mtDNA mutation in ND5 subunit of complex I in two MELAS patients. Ann Neurol, 2001: 49: 106-110.

17. Robbins Basic Pathology. $7^{\text {th }}$ edition. 235-237.

18. Walter Israel, Walter JB, Talbot IC. General Pathology: 7th edition: 66-67. 
19. Magaret S, et al. MELAS: Radiolabeled PCR is necessary for accurate detection of low percentage of mutation. 1997: 82: 2826-2831.

20. Holt I, Harding JA, Morgan- Hughes JA. Deletion of muscle mitochondrial Myopathies. Nature. 1988: 331: 717-719.

21. Wallace DC, Singh G, Lott MT, Hodge JA, Sshurr TG, Lezza AM, Elas JD, Nikoskeelaineu EK, Mitochondrial DNA mutation associated with Leber hereditary optic Neuropathy. Science. 1988: 242: 1427-1430.

\begin{tabular}{|c|c|c|}
\hline Serial number & $\begin{array}{l}\text { Plasma lactate in suspected patients } \\
(\mathrm{mg} / \mathrm{dl})\end{array}$ & $\begin{array}{l}\text { Plasma lactate in control patients } \\
(\mathrm{mg} / \mathrm{dl})\end{array}$ \\
\hline 1 & 24 & 6.7 \\
\hline 2 & 22 & 8.7 \\
\hline 3 & 31 & 9.7 \\
\hline 4 & 19.6 & 9.6 \\
\hline 5 & 26.1 & 7.3 \\
\hline 6 & 29.4 & 8.1 \\
\hline 7 & 33.9 & 13.8 \\
\hline 8 & 40.8 & 14.3 \\
\hline 9 & 24.7 & 8.9 \\
\hline 10 & 7 & 12.5 \\
\hline 11 & 8.1 & 14.8 \\
\hline 12 & 11.4 & 13.8 \\
\hline 13 & 10.5 & 9.4 \\
\hline 14 & 8.7 & 13.5 \\
\hline 15 & 7.8 & 12.8 \\
\hline 16 & 9.7 & 9.8 \\
\hline 17 & 7.4 & 13.6 \\
\hline 18 & 15 & 11.9 \\
\hline 19 & 14.7 & 15.8 \\
\hline 20 & 13.6 & 14.9 \\
\hline
\end{tabular}

